\title{
Modeling the Interrelationship between the Enablers of Cloud Computing
}

\author{
Ambika Devi Amma.T \\ Research Scholar Karpagam \\ University. \\ (Professor, Dept .of Computer \\ Science \&Engg, NSS College \\ of Engineering, Palakkad, \\ Kerala, India 678008
}

\author{
Pramod.V.R, PhD. \\ Associate. Professor, Dept. of \\ Mechanical Engineering, NSS \\ College of Engg, Palakkad, \\ Kerala, India 678008
}

\author{
Radhika.N, PhD. \\ Associate Professor, \\ Computer Science \& Engg \\ Dept, Amrutha University, \\ Coimbatore, TamilNadu, \\ India 641105
}

\begin{abstract}
Interpretive structural modeling (ISM) is a well established methodology for identifying the interrelationship between the variables influencing the system. ISM approach starts with the identification of variables affecting the system. The strategy of cloud computing is subjected to many enablers. This paper deals with the representation of ISM to explicit the interrelationship between the enablers of cloud computing. After listing the enablers a structural self interaction matrix (SSIM) is developed based on pairwise comparison of variables. Transitivity is checked on the reachability matrix obtained from converting SSIM. The enablers are classified into four clusters based on their driver power and dependence. A diagraph is constructed, then ISM model is derived. In this paper key concepts mitigating the most sensitive enablers are discussed in detail.
\end{abstract}

Keywords: ISM, SSIM, driving power, dependence diagraph, cloud computing.

\section{INTRODUCTION}

Cloud computing is an emerging scenario of computing style where applications, datas and resources are provided to users as services over the web. Consumers are not worried about the implementation of the clod but they care about what the service does for them. Cloud computing provides configurable computing resources like servers networks storage and applications as and when required with minimum effort over the internet. The major critical success factor of cloud computing are consistent data availability and security. Data centre facility uses to house computer systems and associated components. It includes redundant power supply, data communication connections and security devices etc. Clouds are location independent providing abstract datacenter. Virtual servers, storage devices and networking are provided without human interaction. A consumer can provision computer facilities like server times and network storage. The cloud capabilities are available over the network and accessed through standard mechanisms like mobile phones laptops and personal digital assistants. Using multitenant model the providers computing resources are pooled to serve multiple customers. The computing resources include storage, processing, memory, network bandwidth and virtual machines. Cloud capabilities can be rapidly, elastically and even automatically sealed out and rapidly released to quickly scale in. By leveraging a metering capacity at some level of abstraction, cloud systems automatically controls and optimize resource use. Transparency is provided for both the provider and consumer regarding the resource usages. The resource usage is monitored, controlled and reported. Standardization and visualization are the keys to reduce infrastructure costs while meeting the dynamic needs of business.

Many organizations are migrating to cloud to derive flexibility and cost reduction benefits from their computing environment. Reasons why cloud computing is popular with business are (a) cost factor,(b)flexibility and (c) service provisioning of the resources. Self service portal allows the clients to help themselves and makes the offering more available from a service perceptive. Automation reduces IT operations costs. Cloud paradigm improves service delivery by applying engineering disciple and scale of economics in an internet inspired architecture.

Cloud deployment models are public cloud, private cloud and hybrid cloud. In public cloud the business rents and pay for what they use on demand. In private cloud the service is on their organization network in its IT environments and delivers servicers to their users only. Hybrid cloud uses the combination of both public and private cloud. Public cloud services are available to customers from a third party service provider via the internet. Public clouds provide cost-effective means to deploy solutions. Companies can use it on demand and with the pay-as-you use policy

Private clouds are deployment made inside the on-premise data centers and run by on site servers. Like public cloud private cloud offers cloud computing environment such as elastic on demand capacity, self service provisioning and service based access. Hybrid cloud is a combination of interoperating public and private clouds. Hybrid models allow elasticity pay- as- you -go pricing, network isolation and secure connectivity just like privately owned data centre. Cloud computing provides massively scalable computing resources from anywhere simplifies service delivery. It provides rapid innovation and dynamic platform for next generation datacenters. 
Some of the features of the cloud are discussed herewith. Self service is one of the important features of the cloud. Using rolebased access control it separate authenticated end-users. Dynamic workload management enables the datacenters with automation. Resource automation establishes secure multitenancy and helps prevent contention in the load aware resource engine. Enabling the feature charge back, show back and metering the transparency in cloud business is attained. Cloud is integrated with the products in the datacenter. It is possible to meter the cloud resource usage. Cloud support most of the operating systems and is possible to vary hardware configuration. Role-based access administrations are used for providing secure multitenancy and bring security to the selfservice cloud portal. The enablers are found out [1][2][6]. and is listed in Table 1.

Table 1: Major enablers of cloud computing

\begin{tabular}{|l|l|l|}
\hline \multicolumn{1}{|c|}{ Enabler } & $\begin{array}{l}\text { Major enabler of cloud } \\
\text { computing }\end{array}$ & References \\
\hline 1 & Data protection & {$[1][2][6]$} \\
\hline 2 & Confidentiality & {$[1][2][6]$} \\
\hline 3 & Data integrity & {$[1][2][6]$} \\
\hline 4 & Data availability & {$[1][2][6]$} \\
\hline 5 & Multitenancy & {$[1][2][6]$} \\
\hline 6 & Operational efficiency & {$[1][2][6]$} \\
\hline 7 & Elasticity & {$[1][2][6]$} \\
\hline 8 & Interoperability & {$[1][2][6]$} \\
\hline 9 & Authorization & {$[1][2][6]$} \\
\hline 10 & Auditing & {$[1][2][6]$} \\
\hline 11 & Guidance & {$[1][2][6]$} \\
\hline 12 & Standards & {$[1][2][6]$} \\
\hline 13 & Data lineage & {$[1][2][6]$} \\
\hline 14 & Data remanence & {$[1][2][6]$} \\
\hline 15 & Data provenance & {$[1][2][6]$} \\
\hline
\end{tabular}

A brief enumeration of the enables are given below

1 Data protection:- Data available in cloud should be protected from external attacks

2 Confidentiality:-All datas should have authentication and authorization for protection also encryption

3 Data integrity: -data should not be changed in an unauthorized manner.

4 Data Availability:- Data should be available to support the operations.

5 Multitenancy:- Sharing of resources and costs across a large pool of customers.

6 Operational Efficiency:- Efficiency of operation depends on the interfaces and web connection, availability of data.

7 Elasticity: -Application can expand and contract on demand

8 Interoperability:- Provision to operate different clouds in different countries.
9 Authorization: -To manage user accounts and various assurance.

10 Auditing;- keeping the account of usage of consumers.

11 Guidance: -Handling identity and access management.

12 Standards:- Standards are defined by governing body or a market lead. It scarce as cloud is concerned. 13 Data lineage:- following the path of data.(mapping application data flows or data path visualization)

14 Data remanence:- is the residual representation of data that has been in some way erased or removed. 15 Data provenance: -Data has integrity and computationally accurate.

\section{LITERATURE SURVEY}

Cloud computing is becoming a well known buzz word now a days. Privacy issues and security problems are pointed out as barriers for users to adopt into cloud computing systems. Users of cloud computing worry about their business informations and critical IT resources in the cloud computing systems which are vulnerable to be attached [1]. Cloud computing allows providers to develop, deploy and run applications that can easily grow in capacity work rapidly without any concern on the properties and the locations of the undergoing infrastructures[2]. Availability is one of the goals of security. It ensure the user to use them at any time at any place. Hardening and redundancy will enhance the availability of the cloud system. Many cloud computing system provide cloud infrastructure and platforms based on virtual machine [Farzad Sabahi,2011] Confidentiality means keeping user's data secret in the cloud system.

Data integrity means prurience the information. No change or no modification by unauthorized users. Access control is another good in security. Access control means to regulate the use of the system including applications, infrastructure and data. Auditing is another phenomena that could be added as an additional layer above the virtualized OS hosted on the virtual machine [7].Secret information of individual users and business are stored and managed by the service providers. Interpretive structural modeling is a well established methodology for identifying relationships among elements present in a complex structures [15]. ISM is an interactive learning process in which set of directly or indirectly related elements are structured into a comprehensive model. For identity relationships among items, the ism methodology can be established. The variables in the specific problem or issue is identified first and then a contextually relevant subordinate relation is taken. Based on pair-wise comparison of variables, a structural self interaction matrix (SSIM) is developed from the element set. Transitivity is checked and a matrix model is obtained. ISM is derived from the partitioning of the element and an extractive of the structural model[10]. In this approach conceptual and computational leverage are exploited to explain the contextual relationship among a set of variables. According to 
Warfield[11] a set of requirement are needed for interpretive structural modeling.

They are : a) Inclusion of scientific elements b) A complex set of relation can be exhibited c) Complex set of relations permits continuous observation, questioning and modification d) Consequence with perceptions and analytical process of the originators e) Public audience can early learn

\section{METHODOLOGY}

ISM is an interpretive methodology go as the judgment of the group decides the relationship of different elements in the system. An overall structure is extracted from the set of elements hence it is structural in the basis of mutual relationships and the overall structure are portrayed in a diagraph model hence it is a modeling technique. The elements order and their direction on the complexity of relationship can be studied with the help of ISM [11 ]. Warfield uses elementary nations of graph theory and Boolean algebra so as to implement a man machine interactive mode.

The steps involved in ISM

1)The relevant elements in the problem/issue is identified. This can be done by interaction the experts or through literature survey.

2)A contextual relationship between the elements are established.3)A structural self interaction matrix (SSIM) is developed transitivity is checked.4)Reachability matrix from SSIM is developed5)Level partition is done6)Reachability matrix is converted into canonical form 7)Based in the relationship diagraph is drawn8)The diagraph is converted into ISM .9)Review the model for conceptual inconsistency and make necessary modifications

3.1: Structural Self Interaction Matrix (SSIM) Structural Self Interaction Matrix develop the contextual relationship among the variables. Contextual relationship of 'leads to' or 'influences' type must be chosen for analyzing the factors. ie. One factor influence another factor. A contextual relationship between the identified elements are following the above factors.

The associated direction of the relationship is questioned based on the contextual relationship for each factor and the existence of a relationship between two factors $(i, j)$. The four symbol used to denote the direction of relationship between two factors (i,j)are the following

$\mathrm{V}$-is used if factor i will influence factor $\mathrm{j}$ [relation from factor $\mathrm{i}$ to factor $\mathrm{j}$ ]

A-factor $\mathrm{j}$ will influence factor $\mathrm{i}$

$\mathrm{X}$-for both direction relations. ie. factors $(\mathrm{i}, \mathrm{j})$ will influence each other

O-factors (i,j)are unrelated (no relation between factors)

Based on the above the SSIM is developed on the basis of the responses of the experts group, SSIM can be finalized. It is shown Table 2
Table 2: structural Self Interaction Matrix (SSIM)

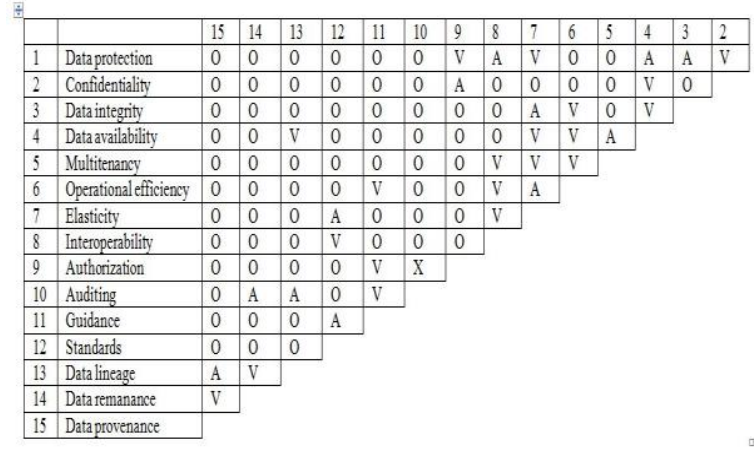

\section{2: Reachability Matrix}

The initial reachability matrix is developed from SSIM. The rules for the matrix construction is as follows (a) if (i,j)entry in SSIM is $\mathrm{V}$, then (i,j) entry in the matrix become I and $(\mathrm{j}, \mathrm{i})$ entry become 0.(b) if the $(I, j)$ entry in SSIM is A, then $(i, j)$ entry in the initial reachability matrix is 0 and $(\mathrm{j}, \mathrm{i})$ entry become 1.(c) if the $(i, j)$ entry in the SSIM is X, then the $(i, j)$ entry become 1 and $(\mathrm{j}, \mathrm{i})$ entry become 1 .(d) if the (i,j) entry in SSIM is $\mathrm{O}$, then the $(\mathrm{i}, \mathrm{j})$ entry become 0 and the $(\mathrm{j}, \mathrm{i})$ entry become 0 . Initial reachability matrix is shown in Table 3 . Final reachability matrix is developed by making use of transitivity property.

Table3: Initial Reachability Matrix

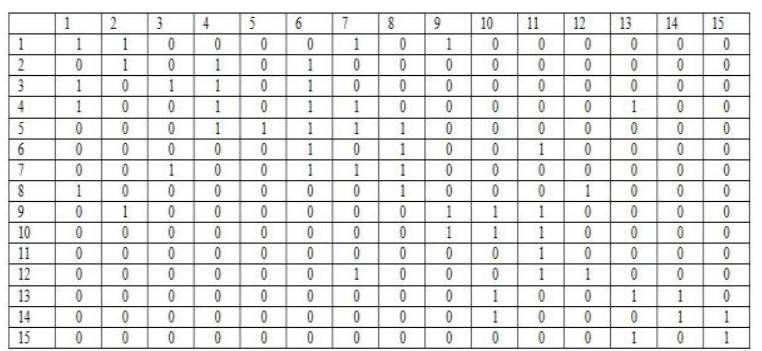

\subsection{Conical Matrix}

From the final reachability matrix the clustering factor in the same level across the rows and columns are considered to develop the conical matrix. The sum of the number of 1 's in the row gives the drive power where as the number of 1 's in the column gives the dependence power [14] [15] [16]. The highest rank of drive power give the maximum number of 1 's into rows and the highest rank of dependence power is calculated by the maximum number of 1's in the columns. Shown in Table 4

Table 4: Final ReachabilityMatrix with driving power and dependence(Conical matrix)

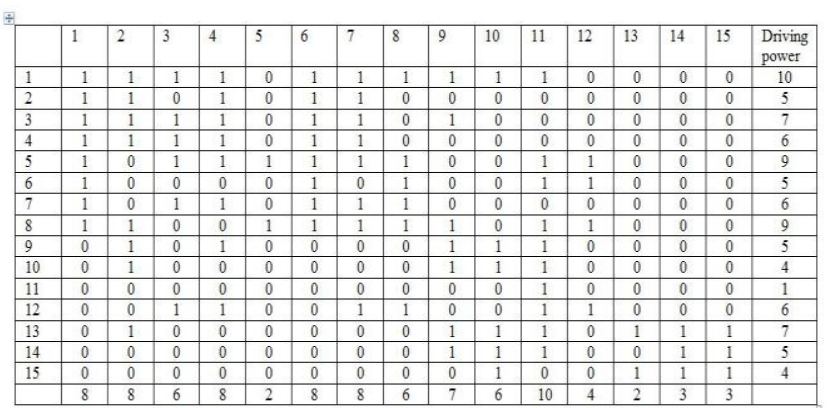




\section{4: Level Partitions}

Level partitions for each factor reachability set and antecedent sets are derived from the final reachability matrix reachability set consist of the factor itself and the factor it may impact . Intersection of these two sets is derived for all the factors. The factors for which the reachability and the intersection sets are the same occupy the highest level (top level) in the hierarchy of ISM Table5 .The top level factors will not lead the other factors above their levels in the hierarchy. Hence once the top level factor is identified it is removed from the sets. The process is related to find out the factors in the next level. It is repeated until the level of each factor is found Table 6 .From the levels the diagraph and the ISM model are build.

Table 5: $1^{\mathrm{ST}}$ Level partition

\begin{tabular}{|l|l|l|l|l|}
\hline Enablers & Reachability set & Antecedent set & Intersection & Level \\
\hline 1 & $1,2,3,4,6,7,8,9,10,11$ & $1,2,3,4,5,6,7,8$ & $1,2,3,4,6,7,8$ & \\
\hline 2 & $1,2,4,6,7$ & $1,2,3,4,8,9,10,13$ & $1,2,4$ & \\
\hline 3 & $1,2,3,4,6,7,9$ & $1,3,4,5,7,12$ & $1,3,4,7$ & \\
\hline 4 & $1,2,3,4,6,7$ & $1,2,3,4,5,7,9,12$ & $1,2,3,4,7$ & \\
\hline 5 & $1,3,4,5,6,7,8,11,12$ & 5,8 & 5,8 & \\
\hline 6 & $1,6,8,11,12$ & $1,2,3,4,5,6,7,8$ & $1,6,8,12$ & \\
\hline 7 & $1,3,4,6,7,8,12$ & $1,2,3,4,5,7,8,12$ & $1,3,4,7,8,12$ & \\
\hline 8 & $1,2,5,6,7,8,9,11,12$ & $1,5,6,7,8,12$ & $1,5,6,7,8,12$ & \\
\hline 9 & $2,4,9,10,11$ & $1,9,10,13,14,15$ & 9,10 & \\
\hline 10 & $2,9,10,11$ & $1,9,10,13,14,15$ & 9,10 & \\
\hline 11 & 11 & $1,5,6,8,9,10,11,12,13,14$ & 11 & 1 \\
\hline 12 & $3,4,7,8,11,12$ & $5,6,7,8,12$ & $7,8,12$ & \\
\hline 13 & $2,9,10,11,13,14,15$ & $13,14,15$ & 13,15 & \\
\hline 14 & $9,10,11,14,15$ & $13,14,15$ & 14,15 & \\
\hline 15 & $10,13,14,15$ & $13,14,15$ & $13,14,15$ & \\
\hline
\end{tabular}

Table 6: levels of enablers

\begin{tabular}{|c|c|c|c|c|}
\hline Enablers & Reachability set & Antecedent set & Intersection & Level \\
\hline 1 & $1,6,12$ & $1,2,3,4,6,7$ & 1,6 & 6 \\
\hline 2 & 2,4 & $2,3,4$ & 2,4 & 8 \\
\hline 3 & 3 & 3 & 3 & 9 \\
\hline 4 & 2,4 & $2,3,4$ & 2,4 & 8 \\
\hline 5 & $1,3,4,5,6,7,8,12$ & 5,8 & 5,8 & 5 \\
\hline 6 & $1,6,12$ & $1,2,3,4,6,7$ & 1,6 & 6 \\
\hline 7 & $3,4,7,12$ & 7,12 & 7,12 & 7 \\
\hline 8 & $1,3,4,5,6,7,8,12$ & 5,8 & 5,8 & 5 \\
\hline 9 & $2,4,9,10$ & $1,3,8,9,10,13,14$ & 9,10 & 2 \\
\hline 10 & $2,4,9,10$ & $1,3,8,9,10,13,14$ & 9,10 & 2 \\
\hline 11 & 11 & $1,5,6,8,9,10,11,12,13,14$ & 11 & 1 \\
\hline 12 & $3,4,7,12$ & 7,12 & 7,12 & 7 \\
\hline 13 & 2,13 & 13 & 13 & 4 \\
\hline 14 & 14,15 & $13,14,15$ & 14,15 & 3 \\
\hline 15 & 14,15 & $13,14,15$ & 14,15 & 3 \\
\hline
\end{tabular}

\section{5: Diagraph Construction}

The preliminary diagraph including transitive links is obtained from the conical form of reachability matrix $[14,15,16]$. Diagraph is used to represent the interdependences of the elements in the terms of nodes and edges. The top level factor is positioned at the top of the diagraph second level in second position and so on until the lowest position is reached Figure1

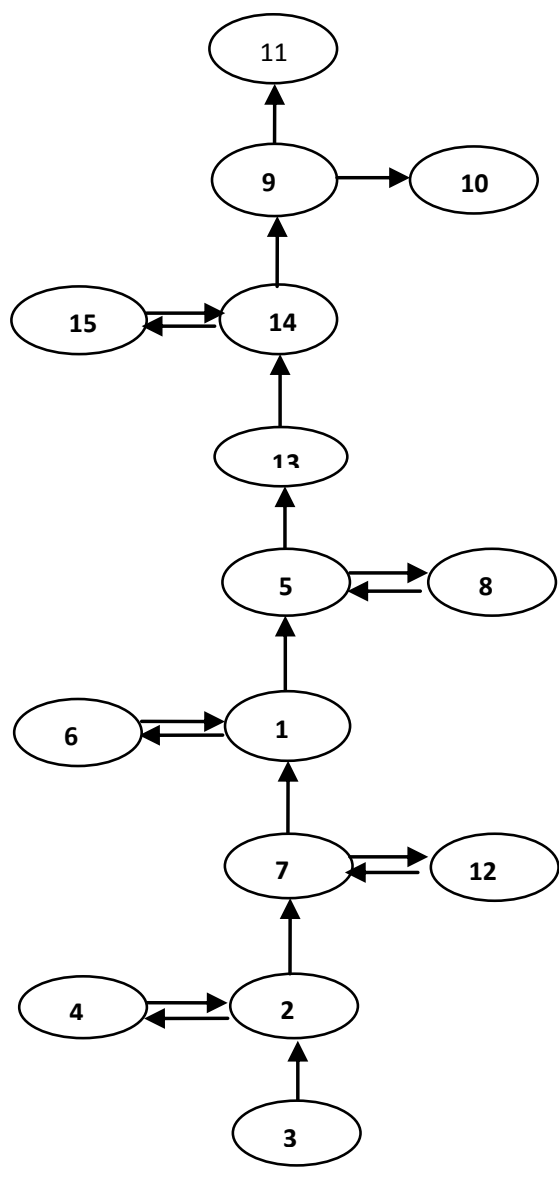

Figure 1. ISM Diagraph

\section{6: Development of ISM from diagraph}

The nodes at the diagraph is replaced with statement for the conversion of diagraph into an ISM model

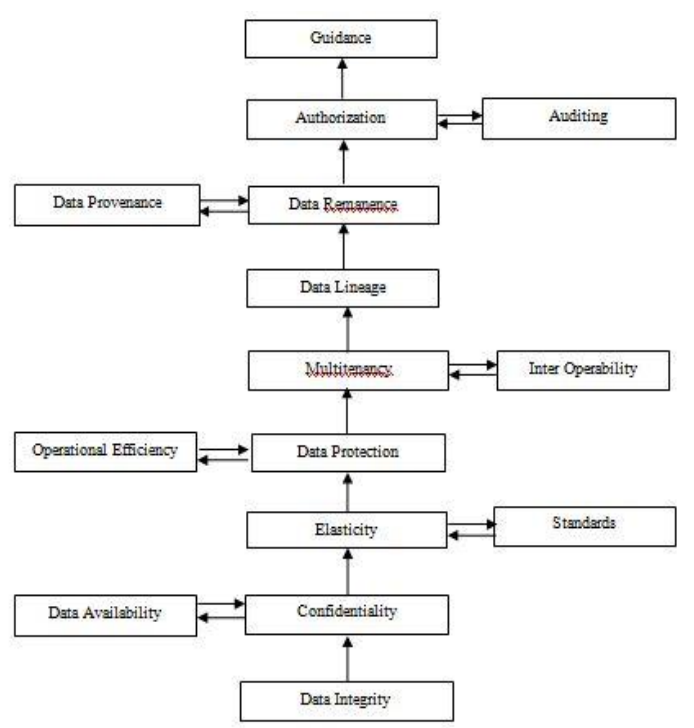

Figure 2. Interpretive Structural Model 


\section{Result \&Discussion}

The drive power and dependence power of factors is analyzed. Analysis is done to identify the key elements which will drive the system in various categories. The elements have been classified into four categories depending upon their drive power and dependence power. They are autonomous, linkage, dependent and independent. Drive power dependence diagram is shown in figure 3

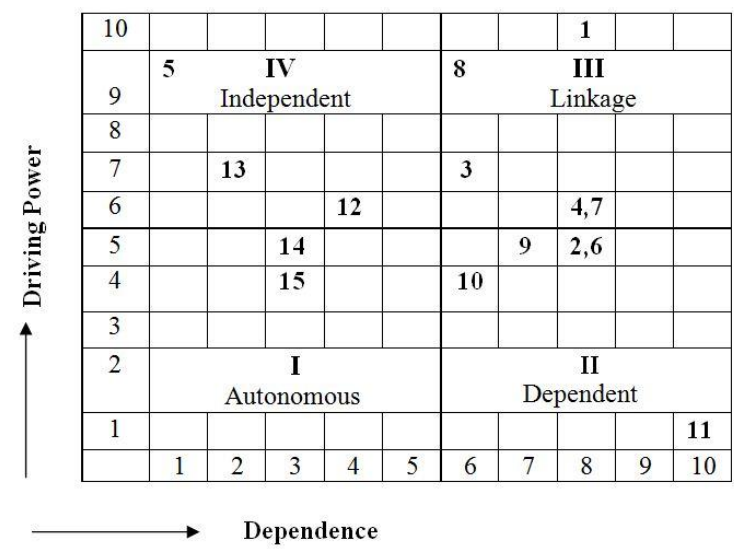

Figure 3 .Driving power Dependence Diagram.

Autonomous elements: They have weak drive power and weak dependence power. They are not strongly connected to the system. We will be able to disconnect it from the system.

Linkages: The elements under the section have strong driving power and strong dependence power. These elements are unstable in the fact that any action on theses elements will have an effect on others and also feedback effect on themselves.

Dependent: These elements have strong dependence power and weak drive power

Independent: These elements have strong drive power and weak dependence power. Elements with strong drive power is the key elements and falls in to the category of independent or linkage section.

5. Conclusion: ISM process evolves the identification of elements and their interrelationship. It can also show the position of rank order of elements in the system. Analysis shows some enablers have weak driving power and dependency. Here data provenance can be disconnected from the structure. The study points out the importance of enablers to the structural flexibility. The levels of partition of elements are important for modeling the enablers of cloud computing. The element data provenance and data remanance are coming under autonomous, they can be disconnected from the system. Some enablers auditing, confidentiality, authorization, operational efficiency and guidance have strong dependence and weak driving power and are coming under dependent section. The enablers data protection, data integrity, data availability, elasticity and interoperability are considered as key elements having strong driving power and strong dependence. The elements multitenancy standards and data lineage have strong driving power and weak dependence. They also influence the system. All the thirteen elements considered are very important. As a future scope, fuzzy ISM can be modeled to study the fuzziness among the interrelationship of elements. There may be large number of variables to a problem. The complexity of ISM methodology may increase as the number of variables are increased. So a limited number of variables are considered. Experts help in selecting the variables and analyzing the driving power and dependence power of the variables . ISM methodology is understandable in the interdisciplinary groups. It is easy to use and communicable and hence it is widely used.

\section{References}

[1] Tim Mather, Subra Kumaraswamy, Shahed Latif, "Cloud Security and Privacy- An Enterprise Perspective on Risks and Compliance" O Reilly

[2] Wang.C and Wulf W. A., "Towards a framework for security measurement", 20th National Information Systems Security Conference, Baltimore, MD, Oct. 1997, pp. 522533.

[3] Savola.R and Abie.H, "Development of measurable security for a distributed messaging system," International Journal on Advances in Security, Vol. 2, No. 4 (2009), 2010 .

[4] Jaquith. A, "Security metrics: replacing fear, uncertainty and doubt,"Addison-Wesley, 2007.

[5] AmbikadeviAmma.T, Dr,Radhika.N Dr. Pramod V.R,'ISM for Analysing the interrelationship between the inhibiters of cloud computing"' International Journal of Computer Applications in Engineering Sciences'VOL II ISSUE III. September 2012.

[6] AmbikadeviAmma.T, Dr,Radhika.N Dr. Pramod V.R,"MCDM Approach for the Adoption of Best Cloud", Inter National Journal of Computer Applications Volume 63-No15,February 2013.

[7] Dr.Kumar Saurabh,"Cloud Computing" Wiley India, second edition.].

[8] Cloud Security Alliance. www.cloudsecurityalliance.org [July 4, 2010].

[9] Mandal.A, Deshmukh.S, Vendor selection using interpretive structural modeling (ism). International Journal of Operations and Production Management, 1994, 14(6): 52-59.

[10] Sage.A, Interpretive Structural Modeling: Methodology for Large-scale Systems, 91-164. McGraw-Hill, New York, 1977.

[11] Warfield.J. Developing interconnection matrices in structural modeling. IEEE Transactionsons on Systems, Man and Cybernetics, 2005, 4(1): 81-67.

[12] Wang.C, "Forrester: A close look at cloud computing security issues," http://www.forrester.com/ securityforum 2009, 2009.

[13] IDC, "It cloud services user survey, pt.2: Top benefits \& challenges," http://blogs.idc.com/ie/?p=210, 2008.

[14] Raj T., Attri R. and Jain V., Modelling the factor affectingflexibility in FMS, International Journal of Industrial and System Engineering, 11(4), 350-374 (2012)

[15] Attri R., Grover S., Dev N. and Kumar D., An ISM approachfor modelling the enablers in the implementation of Tota 
Productive Maintenance (TPM), International Journal SystemAssurance Engineering and Management, DOI:

10.1007/s13198-012-0088-7 (2012

[16] Attri R., Grover S., Dev N. and Kumar D., Analysis ofbarriers of Total Productive Maintenance (TPM) International Journal System Assurance Engineering andManagement, DOI: 10.1007/s13198-010129(2012)

[17] Dwork.C et al., "Differential privacy," LECTURE NOTES IN COMPUTER SCIENCE, vol. 4052, p. 1, 2006.

[18] Dwork.C, "Differential privacy: A survey of results," Lecture Notes in Computer Science, vol. 4978, p. 1, 2008.

[19] Dean. J and Ghemawat.S, "MapReduce: simplified data processing on large clusters," in Proceedings of the 6th conference on Symposium on Opearting Systems Design \& Implementation-Volume 6 table of contents, 2004, pp. $10-10$.

[20] Bardin, J"Security Guidance for Critical Areas of Focus in Cloud Computing," www. cloudsecurityalliance.org/ guidance/csaguide.pdf, 2009.

[21] Hwang, K G. Fox, and Dongarra.J, Distributed Systems and Cloud Computing: Clusters, Grids/P2P, and Internet Clouds, Morgan Kaufmann, to appear, 2010
[22] Nick J, “ Journey to the Private Cloud: Security and Compliance," tech. presentation, EMC, Tsinghua Univ., 25 May 2010.

[23] Rittinghouse J and Ransome.J, Cloud Computing: Implementation, Management and Security, CRC Publisher, 2010

[24] "Gartner Says Cloud Computing Will Be As Influential As E-business". Gartner.com. Retrieved 2010-08-22.

[26] Ravi.V. and Shankar. R. (2005), Analysis of interactions among the barriers of reverse logistics, Technological Forecasting and Social Change, 72(8): 1011-1029.

[27] Thakkar. J.,Kanda.A. and Deshmukh, S.G. (2008), Interpretive Structural Modeling (ISM) of IT-enablers for Indian manufacturing SMEs', Information management \& Computer Security, Vol. 16 No.2, pp. 113-136

[28] Quan Liu, Lu Gao, Ping Lou, "Resource Management Based on Multi-Agent Technology for Cloud Manufacturing, IEEE 2011

[29] Farzad Sabahi, “Cloud Computing Security Threats and Responses” IEEE, 2011.

[30] Craig A Lee, “ A Perspective on Scientific Cloud Computing”, ACM 2010

[31] P. Sasikala, " Cloud Computing: present status and the future implications”, Inderscience Enterprises Ltd. 2011 